\title{
Syncope in the Context of Disorganization of Neural and Glial Network of a Brain
}

\author{
Rosman SV* \\ Physician of functional diagnostics of SBIH, Regional psychoneurological clinic, Russia
}

*Corresponding author: Rosman SV, Physician of functional diagnostics of SBIH, Regional psychoneurological clinic, Tver, Russian Federation, Russia.

Received Date: September 24, 2018

Published Date: October 16, 2018

\begin{abstract}
By means of a new method - dispersion of amplitude-frequency characteristics of an alpha rhythm of EEG the evidence that to a syncope is a consequence of functional disorganization of neural and glial network of a brain with a hypofrontality that can become the reason of the termination of information exchange between an organism and the external environment in the presence of the minimum violations of blood supply which aren't registered at cardiological inspection are produced.
\end{abstract}

Key words: Syncope; Dispersion of alpha rhythm

Abbreviation: NGNB: Neural and Glial Network of a Brain; DAFCAR EEG: Dispersion of Ampli-tude-Frequency Characteristics of Alpha Rhythm of the Electroencephalogram

\section{Review}

To syncope is a syndrome, very widespread in neurologic practice. For example, in Great Britain to a syncope and a collapse (R55 on ICD-10) are the sixth most widespread reason of the emergency hospitalization of adults 65 years in medical hospitals are more senior. The average duration of these hospitalization is 5-17 days that displays a variety of tactics of maintaining to a syncope and availability of certain tests. Only one hospitalization is $74 \%$ of the expenses going for inspection of the patient with a syncope [1].

It is considered that to a syncope is a state at which there is a short-term loss of consciousness caused by decrease in brain blood circulation. The term to "syncope" is often used as a synonym of the concept's "faint", "temporary loss of consciousness", "consciousness switching off". This state connected as with neu-rologic (vegetative and not vegetative), and the cardial reasons. Psychological and mental states are the rea-sons to a pseudo-syncope which it is difficult for syncope to distinguish from true.

Long recidivous syncopal states, for different data, about 12 $48 \%$ of healthy young people and about $6 \%$ el-derly. Results of poll of students (on average 20-year age) which has shown that about $20 \%$ of young men and $50 \%$ of girls at least once in life have tested to a syncope are given in work of W Wieling (2004). J. L. Newton and al. (Newcastle, Great Britain) have revealed that at $20 \%$ of patients with recidivous to a syncope other family member tested syncopal symptoms, having assumed thereby hereditary character.

Researches have shown that the autonomic nervous system plays a leading role in a pathophysiology. There are certificates on existence of dysfunction of the central and peripheral nervous system [2].

Now in all countries the office of treatment of syncope are created to organize in themselves the correspond-ing multidisciplinary skills and experience.

Search of the reasons of these states is run by process of elimination - generally try to find cardiovascular frustration which could lead to sharp violation, for example, of brain blood circulation. And it is much sim-pler to make it in cardiology, than to find significant signs of neurologic frustration in connection with insuf-ficient development of diagnostics of defeat of the NGNB.

At the same time some researchers have reported about high prevalence of alarm, a depression, somatic frus-tration and an alcoholism at patients with not clear to a syncope. Moreover, some authors have found out that at patients with recuring to a syncope 
of various etiology also considerably the quality of life both in physi-cal, and in the psychological relation [3].

At patients with a syncope psychiatric examination is generally carried out by method of the structured in-terview with the psychologist and/or by means of the special questionnaire. The questionnaire of Minnesota Multiphasic Personality Inventory-2 (MMPI-2)-the most widely recognized means for assessment of the psy-chological status of the adult is most often used. After psychological examination clinical diagnoses are for-mulated according to criteria of the Diagnostic and statistical guide to intellectual frustration-IV (Diagnostic and Statistical Manual of Mental Disorders-IV (DSMD-IV).

Some authors have estimated influence of introduction of stationary protocols [2]. These researches have shown that it is possible to improve diagnostics and to optimize inspection. However, many unnecessary researches and hospitalization are still carried out. Thereof costs of inspection and diagnostics have in-creased. The present short publication doesn't apply for the comprehensive research explaining the reasons of the phenomenon in which big institutes are engaged. These are rather notes from practice which allow to look at a problem from the new party process of violation of information exchange in the damaged neural and glial network by means of new, for the present poorly known method - DAFCAR EEG [3].

Really, very small attention is for some reason paid to the fact that a syncopal state - process of violation of information exchange between the person and the environment. According to his success is connected by the previous publications with functional solvency of neural and glial network. It is quite logical to assume that such borderlines of NGNB at which the slightest dysfunction connected with increase in requirements to functional ability of hypermarket leads to his full disorganization and the termination of information ex-change with the external environment are possible.

Before identification of such violations presented insuperable difficulties owing to complexity and high cost of such researches.
However, the researches made in various fields of psychopathology by means of a new method - DAFCAR EEG, have shown that there are correlation communications between psychopathology and disorganization of NGNB, and DAFCAR EEG - a marker of these violations.

For the purpose of identification of violations in NGNB at syncopal violations 47 people more young have been examined than 50 years: men-23, women-24. Middle age: men-32.2 years, women-29.9 years. Investi-gated EEG with standard imposing of electrodes on the international 10-20 system with registration in the background at rest blindly and after hyper ventilating test was carried out. Further the range of EEG was pro-cessed according to the special program [4] and about the help of the statistical Statistica 12.0 program. At all these sick EEG it was carried out with the differential and diagnostic purpose because at cardiological researches (the ECG, ultrasonography) significant violations it was revealed not.

As a result of researches, the following regularities have been revealed.

a) At all patient's various violations of DAFCAR EEG are established to a syncope; in one case there were no normal indicators.

b) The general frequency of an alpha rhythm was in norm limits $-10.0 \pm 0.25 \mathrm{~Hz}$.

c) Alpha rhythm frequency in frontal assignments (F3, F4) was below norm on average on $0.5-0.75 \mathrm{~Hz}$ that demonstrates existence of a functional hypofrontality.

d) The DAFCAR dispersive indexes were much lower than normal values that demonstrates the moder-ated or expressed disorganization of functional ability of NGNB $[3,4]$.

e) These regularities are clearly demonstrated on the given dispersive cartograms. In figure 1 the aver-age cartogram at men is presented. Data are normalized in comparison with DAFCAR EEG cartogram during other researches (Figure 1).

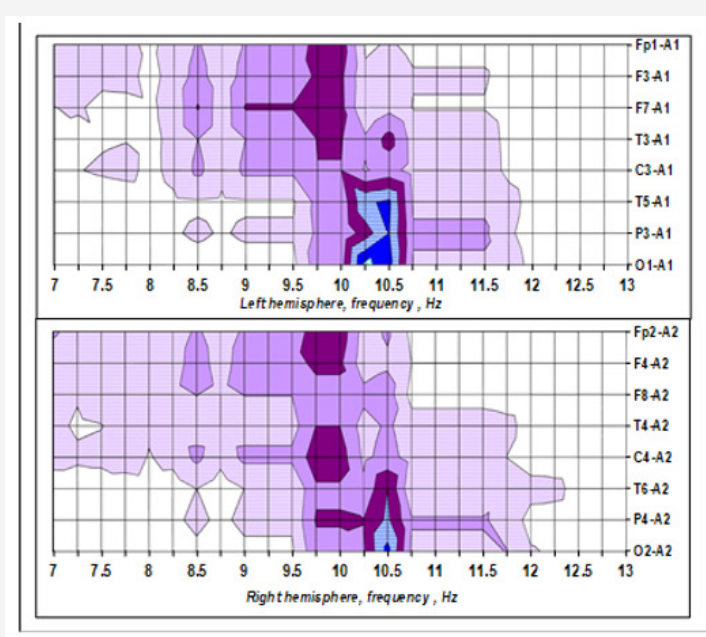

A

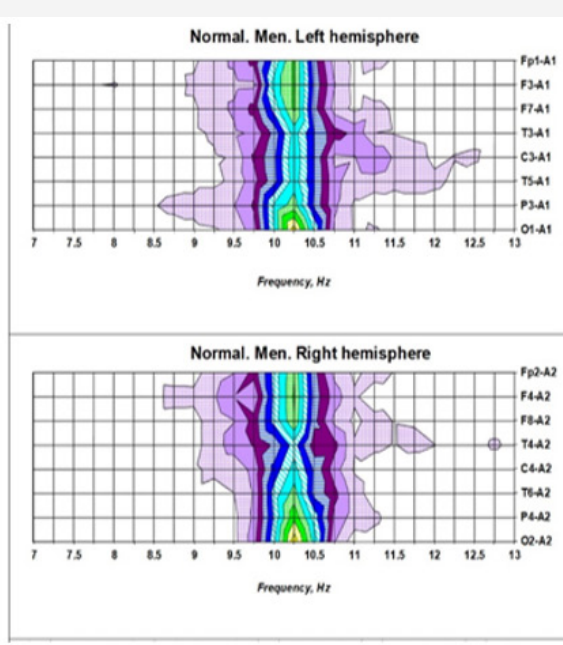

B

Figure 1: Comparative cartograms of DAFCAR EEG at syncopal states (A) is also normal (B). 


\section{Conclusion}

1. Patients with attacks to a syncope have signs of functional disorganization of NGNB.

2. Disorganization of NGNB is shown in a dissynchronization of activity of the cellular elements mak-ing her: in practice considerable splitting of a range of an alpha rhythm of EEG is registered.

3. These changes easily can visually also be registered by means of DAFCAR EEG technique with crea-tion of cartograms and calculation of the DAFCAR indexes.

4. With high degree of probability, it is possible to claim that syncopal states come in result of func-tional inferiority of NGNB, in which even insignificant violations in blood supply of a brain, which normal don't cause any changes can provoke the termination of information exchange with the external environment - loss of orientation in time, space and the personality [5].

\section{References}

1. Alegria JR, Gersh BJ, Scott CG, Hodge DO, Hammill SC, et al. (2003) Comparison of frequency of recurrent syncope after beta-blocker therapy versus conservative man-agement for patients with vasovagal syncope. Amer J Cardiol 92(1): 82-84.

2. Ammirati F, Coliviccbl F, Santini M (200) Diagnosing syncope in the clinical practice. Implementation of a simplified diagnostic algorithm in a multicentre prospective trial -the OESIL 2 study (Osservatorio Epidemiologico della Sincope nei Lazio). Eur Heart J 21: 935-940.

3. Rosman SV (2017) The Theoretical Foundations of Dispersion of Amplitude-Frequency Characteristics of the Al-pha Rhythm of the EEG. Glob J Add \& Rehab Med 2(3): 555587.

4. Rosman SV (2013) Diagnostic capabilities of dispersion mapping the alpha rhythm of the electroencephalogram. Mental health 6: 64-69.

5. Rosman SV (2018) The «System 3+3» in a Problem of Searching of a New Paradigm in Psychiatry. Glob J Add \& Rehab Med. 6(1): 555676. 